\title{
Diffraction of p-Waves by Edge Crack in an Infinite Orthotropic Strip
}

\author{
Aritri Nandi · Prasanta Basak - S. C. Mandal
}

Published online: 23 January 2015

(C) Springer India Pvt. Ltd. 2015

\begin{abstract}
In this paper, the two dimensional interaction of p-waves with an edge crack in an infinite orthotropic strip has been studied. The mixed boundary value problem of determining the unknown stress distribution outside the crack has been converted to the determination of the solution of a Fredholm integral equation of second kind by Fourier transform. Stress intensity factor (SIF) at the tip of the crack has been calculated by solving integral equation numerically and plotted to show the effect of strip width on SIF. Finally normal stress at distant points from the crack has been calculated and plotted for various parameter to show the effect of material orthotropy.
\end{abstract}

Keywords In-plane $\cdot$ Diffraction $\cdot$ Edge crack $\cdot$ Stress intensity factor $\cdot$ Strip

\section{Introduction}

Recently, the study of interaction of elastic waves with cracks has been gained much importance in view of increased usage of macroscopically anisotropic construction materials such as fibre-reinforced composites. Problems involving one or more cracks in infinite anisotropic medium has been studied by many authors. Studies of Griffith crack problem in orthotropic medium has been made by Cinar and Erdogan [1], Satapathy and Parhi [2], Piva and Viola [3], Kassir and Tse [4] and Dhaliwal [5]. Das and Debnath [6] solved the problem of determining the stress intensity factor (SIF) at the tips of the Griffith crack in a punched layer by reducing the problem to the solution of simultaneous integral equations, which have been solved by using Tchebyshev polynomials.

Finite crack perpendicular to the surface of the infinitely long elastic strip has been studied by Chen [7] for an impact load and by Srivastava et al. [8] for normally incident waves. Shindo et al. [9] analyzed the problem of impact response of a finite crack in an orthotropic strip. The diffraction of elastic waves by three coplanar Griffith Cracks in an orthotropic medium has

A. Nandi · P. Basak · S. C. Mandal (凶)

Department of Mathematics, Jadavpur University, Kolkata 700032, West Bengal, India

e-mail: scmandal.ju@gmail.com; scmandal@math.jdvu.ac.in 
been studied by Sarkar et al. [10]. Munshi and Mandal [11] solved the problem of diffraction of p-waves by edge crack in an infinitely long elastic strip. Das et al. [12] addressed the problem of symmetric edge cracks in an orthotropic strip under normal loading by seeking the solution of a pair of simultaneous integral equations with Cauchy type singularities. Das [13] presented the expression of SIF using weight function of an edge crack in an infinite orthotropic strip. Edge crack under normal loading studied by Das et al. $[14,15]$ in the surface of an orthotropic strip bonded to an orthotropic half plane and in the surface of an orthotropic strip of finite thickness bonded to another orthotropic finite strip.

In the present paper the interaction of p-wave with edge crack in an orthotropic strip has been analyzed. Fourier transform technique is used to reduce the boundary value problem to the solution of a pair of dual integral equations. Using Abel's transform the problem has been further reduced to the solution of a Fredholm integral equation of second kind. The dynamic SIF is computed for several fibre-reinforced composite materials and numerical values have been shown by means of graph for various geometrical parameters. Normal stress outside the crack has also been calculated and plotted as 3D graphs for various parameters.

\section{Formulation of the Problem}

Let us consider the diffraction of normally incident longitudinal wave by an edge crack in an infinitely long orthotropic strip of width $h_{1}$. Location of the crack is defined by $0 \leq x_{1} \leq$ $a,-\infty<z_{1}<\infty, y_{1}=0$. Normalizing all the lengths with respect to 'a' and putting $\frac{x_{1}}{a}=$ $x, \frac{y_{1}}{a}=y, \frac{z_{1}}{a}=z, \frac{h_{1}}{a}=h$, the edge crack is defined by $0 \leq x \leq 1,-\infty<z<\infty, y=0$ (Fig. 1) referred to Cartesian co-ordinate axes $(x, y, z)$. Let the time harmonic wave travels in the direction of the positive $y$ axis.

Displacement components are also made dimensionless with respect to ' $a$ '. The dimensionless displacement components in $x, y$ directions are assumed to be $u, v$ respectively and are given by

$$
u=u(x, y, t) \text { and } v=v(x, y, t)
$$

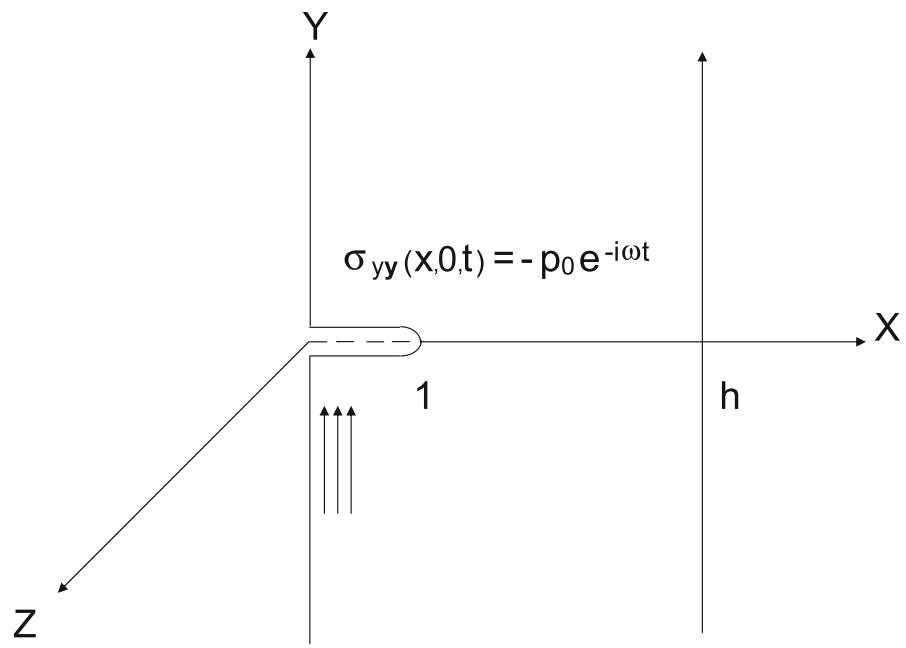

Fig. 1 Crack geometry 
The nonzero stress components $\sigma_{x x}, \sigma_{y y}, \sigma_{x y}$ are

$$
\begin{aligned}
\sigma_{x x} / \mu_{12} & =C_{11} \frac{\partial u}{\partial x}+C_{12} \frac{\partial v}{\partial y} \\
\sigma_{y y} / \mu_{12} & =C_{12} \frac{\partial u}{\partial x}+C_{22} \frac{\partial v}{\partial y} \\
\sigma_{x y} / \mu_{12} & =\frac{\partial u}{\partial y}+\frac{\partial v}{\partial x}
\end{aligned}
$$

and $C_{i j}(\mathrm{i}, \mathrm{j}=1,2)$ are non-dimensional parameters related to the elastic constants by the relations

$$
\begin{aligned}
& C_{11}=E_{1} / \mu_{12}\left(1-v_{12}^{2} E_{2} / E_{1}\right) \\
& C_{22}=E_{2} / \mu_{12}\left(1-v_{12}^{2} E_{2} / E_{1}\right)=C_{11} E_{2} / E_{1} \\
& C_{12}=v_{12} E_{2} / \mu_{12}\left(1-v_{12}^{2} E_{2} / E_{1}\right)=v_{12} C_{22}=v_{21} C_{11}
\end{aligned}
$$

where $E_{i}, \mu_{i j}$ and $v_{i j}(\mathrm{i}, \mathrm{j}=1,2,3)$ denote the engineering elastic constants of the material and satisfy Maxwell's relation:

$$
v_{i j} / E_{i}=v_{j i} / E_{j}
$$

The displacement equations of motion for orthotropic material are

$$
\begin{aligned}
& C_{11} \frac{\partial^{2} u}{\partial x^{2}}+\frac{\partial^{2} u}{\partial y^{2}}+\left(1+C_{12}\right) \frac{\partial^{2} v}{\partial x \partial y}=\frac{a^{2}}{c_{s}^{2}} \frac{\partial^{2} u}{\partial t^{2}} \\
& C_{22} \frac{\partial^{2} v}{\partial y^{2}}+\frac{\partial^{2} v}{\partial x^{2}}+\left(1+C_{12}\right) \frac{\partial^{2} u}{\partial x \partial y}=\frac{a^{2}}{c_{s}^{2}} \frac{\partial^{2} v}{\partial t^{2}}
\end{aligned}
$$

Substitution of $u(x, y, t)=u(x, y) e^{-i \omega t}$ and $v(x, y, t)=v(x, y) e^{-i \omega t}$ in Eqs. (9) and (10) reduces them to

$$
\begin{aligned}
& C_{11} \frac{\partial^{2} u}{\partial x^{2}}+\frac{\partial^{2} u}{\partial y^{2}}+\left(1+C_{12}\right) \frac{\partial^{2} v}{\partial x \partial y}+k_{s}^{2} u=0 \\
& C_{22} \frac{\partial^{2} v}{\partial y^{2}}+\frac{\partial^{2} v}{\partial x^{2}}+\left(1+C_{12}\right) \frac{\partial^{2} u}{\partial x \partial y}+k_{s}^{2} v=0
\end{aligned}
$$

with $k_{s}^{2}=a^{2} \omega^{2} / c_{s}^{2}$, which are to be solved subject to the boundary conditions

$$
\begin{array}{ll}
\sigma_{y y}(x, 0)=-p_{0} & (0 \leq x \leq 1) \\
v(x, 0)=0 & (1 \leq x \leq h) \\
\sigma_{x y}(x, 0)=0 & (0 \leq x \leq h) \\
\sigma_{x y}(0, y)=0=\sigma_{x y}(h, y) \\
\sigma_{x x}(0, y)=0=\sigma_{x x}(h, y)
\end{array}
$$

where $p_{0}$ is a constant.

The term $e^{-i \omega t}$ which is common to all field variables is omitted in the sequel.

In absence of boundary, we assume the solution of the problem as $u(x, y)=f(y) e^{i \xi x}$ and $v(x, y)=g(y) e^{i \xi x}$, where $f(y)$ and $g(y)$ are unknown functions to be determined. From Eqs. (11) and (12) it can be shown that both $f(y)$ and $g(y)$ satisfy a biquadratic equation whose trial solutions are taken as $f(y)=e^{-\gamma y}$ and $g(y)=e^{-\gamma y}$. Then $\gamma$ satisfies the following biquadratic equation 


$$
C_{22} \gamma^{4}+\left\{\left(C_{12}^{2}+2 C_{12}-C_{11} C_{22}\right) \xi^{2}+\left(1+C_{22}\right) k_{s}^{2}\right\} \gamma^{2}+\left(k_{s}^{2}-\xi^{2}\right)\left(k_{s}^{2}-C_{11} \xi^{2}\right)=0
$$

and corresponding solution of the Eqs. (11) and (12) are

$$
\begin{aligned}
& u(x, y)=\int_{-\infty}^{\infty}\left[A_{1}(\xi) e^{-\gamma_{1} y}+A_{2}(\xi) e^{-\gamma_{2} y}\right] e^{i \xi x} d \xi \\
& v(x, y)=\int_{-\infty}^{\infty}\left[\alpha_{1} A_{1}(\xi) e^{-\gamma_{1} y}+\alpha_{2} A_{2}(\xi) e^{-\gamma_{2} y}\right] \xi^{-1} e^{i \xi x} d \xi
\end{aligned}
$$

where

$$
\alpha_{j}=\frac{\gamma_{j}^{2}-C_{11} \xi^{2}+k_{s}^{2}}{i \gamma_{j}\left(1+C_{12}\right)} \quad(j=1,2), \quad i=\sqrt{-1}
$$

and $\gamma_{1}^{2}$ and $\gamma_{2}^{2}$ are the roots of the Eq. (18).

Now, if the boundary is present, we consider the solutions as $u(x, y)=f_{1}(x) \cos (\zeta y)$ and $v(x, y)=g_{1}(x) \sin (\zeta y)$ and proceeding similarly as before we obtain the following solutions of Eqs. (11) and (12):

$$
\begin{aligned}
& u(x, y)=\int_{-\infty}^{\infty}\left[\zeta \beta_{1}\left\{D_{1}(\zeta) e^{\gamma_{3} x}-D_{2}(\zeta) e^{-\gamma_{3} x}\right\}+\zeta \beta_{2}\left\{D_{3}(\zeta) e^{\gamma_{4} x}-D_{4}(\zeta) e^{-\gamma_{4} x}\right\}\right] \cos \zeta y d \zeta \\
& v(x, y)=\int_{-\infty}^{\infty}\left[D_{1}(\zeta) e^{\gamma_{3} x}+D_{2}(\zeta) e^{-\gamma_{3} x}+D_{3}(\zeta) e^{\gamma_{4} x}+D_{4}(\zeta) e^{-\gamma_{4} x}\right] \sin \zeta y d \zeta
\end{aligned}
$$

where

$$
\begin{aligned}
& \beta_{1}=\frac{\left(1+C_{12}\right) \gamma_{3}}{\zeta^{2}-C_{11} \gamma_{3}^{2}-k_{s}^{2}} \\
& \beta_{2}=\frac{\left(1+C_{12}\right) \gamma_{4}}{\zeta^{2}-C_{11} \gamma_{4}^{2}-k_{s}^{2}}
\end{aligned}
$$

and $\gamma_{3}^{2}, \gamma_{4}^{2}$ are the roots of the equation

$$
C_{11} \gamma^{4}+\left\{\left(C_{12}^{2}+2 C_{12}-C_{11} C_{22}\right) \zeta^{2}+\left(1+C_{11}\right) k_{s}^{2}\right\} \gamma^{2}+\left(k_{s}^{2}-\zeta^{2}\right)\left(k_{s}^{2}-C_{22} \zeta^{2}\right)=0
$$

Therefore using boundary condition (15), the combined solutions of Eqs. (11) and (12) may be written as

$$
\begin{aligned}
u(x, y)= & \int_{-\infty}^{\infty}\left[e^{-\gamma_{1} y}+\beta e^{-\gamma_{2} y}\right] A_{1}(\xi) e^{i \xi x} d \xi+\int_{0}^{\infty}\left[\zeta \beta_{1}\left\{D_{1}(\zeta) e^{\gamma_{3} x}-D_{2}(\zeta) e^{-\gamma_{3} x}\right\}\right. \\
& \left.+\zeta \beta_{2}\left\{D_{3}(\zeta) e^{\gamma_{4} x}-D_{4}(\zeta) e^{-\gamma_{4} x}\right\}\right] \cos (\zeta y) d \zeta \\
v(x, y)= & \int_{-\infty}^{\infty}\left[\alpha_{1} e^{-\gamma_{1} y}+\beta \alpha_{2} e^{-\gamma_{2} y}\right] \xi^{-1} A_{1}(\xi) e^{i \xi x} d \xi+\int_{0}^{\infty}\left[D_{1}(\zeta) e^{\gamma_{3} x}+D_{2}(\zeta) e^{-\gamma_{3} x}\right. \\
& \left.+D_{3}(\zeta) e^{\gamma_{4} x}+D_{4}(\zeta) e^{-\gamma_{4} x}\right] \sin (\zeta y) d \zeta
\end{aligned}
$$

and the stress components are given by

$$
\begin{aligned}
\sigma_{x x} / \mu_{12}= & \int_{-\infty}^{\infty}\left[\left(i \xi^{2} C_{11}-\alpha_{1} \gamma_{1} C_{12}\right) e^{-\gamma_{1} y}+\left(i \xi^{2} C_{11}-\alpha_{2} \gamma_{2} C_{12}\right) \beta e^{-\gamma_{2} y}\right] \xi^{-1} A_{1}(\xi) e^{i \xi x} d \xi \\
& +\int_{0}^{\infty}\left[\left(C_{11} \beta_{1} \gamma_{3}+C_{12}\right)\left\{D_{1}(\zeta) e^{\gamma_{3} x}+D_{2}(\zeta) e^{-\gamma_{3} x}\right\}\right.
\end{aligned}
$$




$$
\begin{aligned}
& \left.+\left(C_{11} \beta_{2} \gamma_{4}+C_{12}\right)\left\{D_{3}(\zeta) e^{\gamma_{4} x}+D_{4}(\zeta) e^{-\gamma_{4} x}\right\}\right] \zeta \cos (\zeta y) d \zeta \\
\sigma_{y y} / \mu_{12}= & \int_{-\infty}^{\infty}\left[\left(i \xi^{2} C_{12}-\alpha_{1} \gamma_{1} C_{22}\right) e^{-\gamma_{1} y}+\left(i \xi^{2} C_{12}-\alpha_{2} \gamma_{2} C_{22}\right) \beta e^{-\gamma_{2} y}\right] \xi^{-1} A_{1}(\xi) e^{i \xi x} d \xi \\
& +\int_{0}^{\infty}\left[\left(C_{12} \beta_{1} \gamma_{3}+C_{22}\right)\left\{D_{1}(\zeta) e^{\gamma_{3} x}+D_{2}(\zeta) e^{-\gamma_{3} x}\right\}\right. \\
& \left.+\left(C_{12} \beta_{2} \gamma_{4}+C_{22}\right)\left\{D_{3}(\zeta) e^{\gamma_{4} x}+D_{4}(\zeta) e^{-\gamma_{4} x}\right\}\right] \zeta \cos (\zeta y) d \zeta \\
\sigma_{x y} / \mu_{12}= & \int_{-\infty}^{\infty}\left[\left(i \alpha_{1}-\gamma_{1}\right) e^{-\gamma_{1} y}+\left(i \alpha_{2}-\gamma_{2}\right) \beta e^{-\gamma_{2} y}\right] A_{1}(\xi) e^{i \xi x} d \xi \\
& +\int_{0}^{\infty}\left[\left(\gamma_{3}-\zeta^{2} \beta_{1}\right) D_{1}(\zeta) e^{\gamma_{3} x}+\left(\zeta^{2} \beta_{1}-\gamma_{3}\right) D_{2}(\zeta) e^{-\gamma_{3} x}\right. \\
& \left.+\left(\gamma_{4}-\zeta^{2} \beta_{2}\right) D_{3}(\zeta) e^{\gamma_{4} x}+\left(\zeta^{2} \beta_{2}-\gamma_{4}\right) D_{4}(\zeta) e^{-\gamma_{4} x}\right] \sin (\zeta y) d \zeta
\end{aligned}
$$

where $A_{1}(\xi), D_{1}(\zeta), D_{2}(\zeta), D_{3}(\zeta)$ and $D_{4}(\zeta)$ are the unknown functions of transformed variables $\xi$ and $\zeta$ which are to be determined from the boundary conditions and $\beta$ is given by

$$
\beta=\frac{\gamma_{1}-i \alpha_{1}}{i \alpha_{2}-\gamma_{2}}
$$

With the aid of the boundary conditions (13)-(14), we obtain the following dual integral equations for the unknown function $A(\xi)$

$$
\begin{aligned}
& \int_{-\infty}^{\infty} A(\xi) e^{i \xi x} d \xi=0 \quad(1 \leq x \leq h) \\
& \int_{-\infty}^{\infty} H(\xi) A(\xi) e^{i \xi x} d \xi=-\mu_{12}^{-1} p_{0}+Q(x) \quad(0 \leq x \leq 1)
\end{aligned}
$$

where

$$
\begin{aligned}
A(\xi)= & \frac{\left(\alpha_{1}+\beta \alpha_{2}\right) A_{1}(\xi)}{\xi} \\
H(\xi)= & \frac{i C_{12} \xi^{2}-C_{22} \alpha_{1} \gamma_{1}+\beta\left(i C_{12} \xi^{2}-C_{22} \alpha_{2} \gamma_{2}\right)}{\alpha_{1}+\beta \alpha_{2}} \\
Q(x)=- & \int_{0}^{\infty}\left[\left(C_{12} \beta_{1} \gamma_{3}+C_{22}\right)\left\{D_{1}(\zeta) e^{\gamma_{3} x}+D_{2}(\zeta) e^{-\gamma_{3} x}\right\}\right. \\
& \left.+\left(C_{12} \beta_{2} \gamma_{4}+C_{22}\right)\left\{D_{3}(\zeta) e^{\gamma_{4} x}+D_{4}(\zeta) e^{-\gamma_{4} x}\right\}\right] \zeta d \zeta
\end{aligned}
$$

\section{Solution}

The solution of the integral Eqs. (25) and (26) is taken in the form

$$
A(\xi)=\frac{a_{0}}{2 \theta} \int_{0}^{1} \operatorname{tg}(t) J_{0}(\xi t) d t
$$

so that the Eq. (25) is automatically satisfied and Eq. (26) can be written as

$$
\int_{0}^{1} \operatorname{tg}(t) \int_{0}^{\infty}\left[H_{1}(\xi)+\xi\right] J_{0}(\xi t) \cos (\xi x) d \xi d t=-\frac{\mu_{12}^{-1} p_{0}}{a_{0}}+\frac{Q(x)}{a_{0}}
$$


where

$$
\begin{aligned}
& H_{1}(\xi)=\frac{H(\xi)}{\theta}-\xi \longrightarrow 0 \text { as } \xi \longrightarrow \infty \\
& \theta=\frac{\left[\left(C_{12}^{2}+C_{12}-C_{11} C_{22}\right)\left(C_{12} N_{1} N_{2}-C_{11}\right)-C_{22}\left\{C_{11}\left(N_{1}^{2}+N_{1} N_{2}+N_{2}^{2}\right)+C_{12} N_{1}^{2} N_{2}^{2}\right\}\right]}{C_{11}\left(N_{1}+N_{2}\right)\left(1+C_{12}\right)} \\
& N_{1}^{2}=\frac{-\left(C_{12}^{2}+2 C_{12}-C_{11} C_{22}\right)+\sqrt{\left(C_{12}^{2}+2 C_{12}-C_{11} C_{22}\right)^{2}-4 C_{11} C_{22}}}{2 C_{22}} \\
& N_{2}^{2}=\frac{-\left(C_{12}^{2}+2 C_{12}-C_{11} C_{22}\right)-\sqrt{\left(C_{12}^{2}+2 C_{12}-C_{11} C_{22}\right)^{2}-4 C_{11} C_{22}}}{2 C_{22}}
\end{aligned}
$$

and $J_{0}()$ is the Bessel function of first kind of order zero.

Using Abel's transform in Eq. (28) and manipulating, we obtain the following Fredholm integral equation of second kind

$$
g(t)+\int_{0}^{1} u g(u) L_{1}(u, t) d u=R(t)
$$

where

$$
\begin{aligned}
L_{1}(u, t) & =\int_{0}^{\infty} H_{1}(\xi) J_{0}(\xi u) J_{0}(\xi t) d \xi \\
R(t) & =-\frac{\mu_{12}^{-1} p_{0}}{a_{0}}+\frac{2}{\pi a_{0}} \int_{0}^{t} \frac{Q(x) d x}{\sqrt{t^{2}-x^{2}}}
\end{aligned}
$$

Substituting $\zeta D_{1}(\zeta)=E_{1}(\zeta), \zeta D_{2}(\zeta)=E_{2}(\zeta), \zeta D_{3}(\zeta)=E_{3}(\zeta), \zeta D_{4}(\zeta)=E_{4}(\zeta)$, we get from the boundary conditions (16)-(17), the unknown coefficients $E_{1}(\zeta), E_{2}(\zeta)$, $E_{3}(\zeta)$ and $E_{4}(\zeta)$, which can be found to be related to $A(\xi)$ as

$$
\begin{aligned}
& M(\zeta) E_{1}(\zeta) \\
& =2\left(C_{11} \beta_{2} \gamma_{4}+C_{12}\right)\left[\left(\zeta^{-1} \gamma_{3}-\zeta \beta_{1}\right)\left(C_{11} \beta_{2} \gamma_{4}+C_{12}\right) \sinh \left(\gamma_{4} h\right)\left\{e^{-\gamma_{3} h} q_{1}(\zeta)-q_{2}(\zeta)\right\}\right. \\
& +\left(\zeta^{-1} \gamma_{4}-\zeta \beta_{2}\right)\left(C_{11} \beta_{1} \gamma_{3}+C_{12}\right)\left\{\left(e^{-\gamma_{3} h} \cosh \left(\gamma_{4} h\right)-1\right) q_{1}(\zeta)\right. \\
& \left.\left.+\left(\cosh \left(\gamma_{4} h\right)-e^{-\gamma_{3} h}\right) q_{2}(\zeta)\right\}\right]+2\left(\zeta^{-1} \gamma_{4}-\zeta \beta_{2}\right)\left[\left(\zeta^{-1} \gamma_{4}\right.\right. \\
& \left.-\zeta \beta_{2}\right)\left(C_{11} \beta_{1} \gamma_{3}+C_{12}\right) \sinh \left(\gamma_{4} h\right)\left\{e^{-\gamma_{3} h} q_{3}(\zeta)+q_{4}(\zeta)\right\} \\
& +\left(\zeta^{-1} \gamma_{3}-\zeta \beta_{1}\right)\left(C_{11} \beta_{2} \gamma_{4}+C_{12}\right)\left\{\left(e^{-\gamma_{3} h} \cosh \left(\gamma_{4} h\right)-1\right) q_{3}(\zeta)\right. \\
& \left.\left.+\left(\cosh \left(\gamma_{4} h\right)-e^{-\gamma_{3} h}\right) q_{4}(\zeta)\right\}\right] \\
& M(\zeta) E_{2}(\zeta) \\
& =2\left(C_{11} \beta_{2} \gamma_{4}+C_{12}\right)\left[\left(\zeta^{-1} \gamma_{3}-\zeta \beta_{1}\right)\left(C_{11} \beta_{2} \gamma_{4}+C_{12}\right) \sinh \left(\gamma_{4} h\right)\left\{e^{\gamma_{3} h} q_{1}(\zeta)-q_{2}(\zeta)\right\}\right. \\
& +\left(\zeta^{-1} \gamma_{4}-\zeta \beta_{2}\right)\left(C_{11} \beta_{1} \gamma_{3}+C_{12}\right)\left\{\left(1-e^{\gamma_{3} h} \cosh \left(\gamma_{4} h\right)\right) q_{1}(\zeta)+\left(e^{\gamma_{3} h}\right.\right. \\
& \left.\left.\left.-\cosh \left(\gamma_{4} h\right)\right) q_{2}(\zeta)\right\}\right]+2\left(\zeta^{-1} \gamma_{4}-\zeta \beta_{2}\right)\left[\left(\zeta^{-1} \gamma_{4}\right.\right. \\
& \left.-\zeta \beta_{2}\right)\left(C_{11} \beta_{1} \gamma_{3}+C_{12}\right) \sinh \left(\gamma_{4} h\right)\left\{q_{4}(\zeta)-e^{\gamma_{3} h} q_{3}(\zeta)\right\} \\
& +\left(\zeta^{-1} \gamma_{3}-\zeta \beta_{1}\right)\left(C_{11} \beta_{2} \gamma_{4}+C_{12}\right)\left\{\left(e^{\gamma_{3} h} \cosh \left(\gamma_{4} h\right)-1\right) q_{3}(\zeta)\right. \\
& \left.\left.+\left(\cosh \left(\gamma_{4} h\right)-e^{\gamma_{3} h}\right) q_{4}(\zeta)\right\}\right] \\
& M(\zeta) E_{3}(\zeta) \\
& =2\left(C_{11} \beta_{1} \gamma_{3}+C_{12}\right)\left[\left(\zeta^{-1} \gamma_{4}-\zeta \beta_{2}\right)\left(C_{11} \beta_{1} \gamma_{3}+C_{12}\right) \sinh \left(\gamma_{3} h\right)\left\{e^{-\gamma_{4} h} q_{1}(\zeta)-q_{2}(\zeta)\right\}\right. \\
& \left.-\left(\zeta^{-1} \gamma_{3}-\zeta \beta_{1}\right)\left(C_{11} \beta_{2} \gamma_{4}+C_{12}\right)\left\{\left(1-e^{-\gamma_{4} h} \cosh \left(\gamma_{3} h\right)\right) q_{1}(\zeta)-\left(\cosh \left(\gamma_{3} h\right)-e^{-\gamma_{4} h}\right) q_{2}(\zeta)\right\}\right] \\
& +2\left(\zeta^{-1} \gamma_{3}-\zeta \beta_{1}\right)\left[\left(\zeta^{-1} \gamma_{3}-\zeta \beta_{1}\right)\left(C_{11} \beta_{2} \gamma_{4}+C_{12}\right) \sinh \left(\gamma_{3} h\right)\left\{e^{-\gamma_{4} h} q_{3}(\zeta)+q_{4}(\zeta)\right\}\right.
\end{aligned}
$$


$-\left(\zeta^{-1} \gamma_{4}-\zeta \beta_{2}\right)\left(C_{11} \beta_{1} \gamma_{3}+C_{12}\right)\left\{\left(1-e^{-\gamma_{4} h} \cosh \left(\gamma_{3} h\right)\right) q_{3}(\zeta)\right.$

$\left.\left.-\left(\cosh \left(\gamma_{3} h\right)-e^{-\gamma_{4} h}\right) q_{4}(\zeta)\right\}\right]$

$M(\zeta) E_{4}(\zeta)$

$$
\begin{aligned}
= & 2\left(C_{11} \beta_{1} \gamma_{3}+C_{12}\right)\left[\left(\zeta^{-1} \gamma_{4}-\zeta \beta_{2}\right)\left(C_{11} \beta_{1} \gamma_{3}+C_{12}\right) \sinh \left(\gamma_{3} h\right)\left\{e^{\gamma_{4} h} q_{1}(\zeta)-q_{2}(\zeta)\right\}\right. \\
& +\left(\zeta^{-1} \gamma_{3}-\zeta \beta_{1}\right)\left(C_{11} \beta_{2} \gamma_{4}+C_{12}\right)\left\{\left(1-e^{\gamma_{4} h} \cosh \left(\gamma_{3} h\right)\right) q_{1}(\zeta)\right. \\
& \left.\left.+\left(e^{\gamma_{4} h}-\cosh \left(\gamma_{3} h\right)\right) q_{2}(\zeta)\right\}\right] \\
& +2\left(\zeta^{-1} \gamma_{3}-\zeta \beta_{1}\right)\left[\left(\zeta^{-1} \gamma_{3}-\zeta \beta_{1}\right)\left(C_{11} \beta_{2} \gamma_{4}+C_{12}\right) \sinh \left(\gamma_{3} h\right)\left\{q_{4}(\zeta)-e^{\gamma_{4} h} q_{3}(\zeta)\right\}\right. \\
& -\left(\zeta^{-1} \gamma_{4}-\zeta \beta_{2}\right)\left(C_{11} \beta_{1} \gamma_{3}+C_{12}\right)\left\{\left(1-e^{\gamma_{4} h} \cosh \left(\gamma_{3} h\right)\right) q_{3}(\zeta)\right. \\
& \left.\left.+\left(e^{\gamma_{4} h}-\cosh \left(\gamma_{3} h\right)\right) q_{4}(\zeta)\right\}\right]
\end{aligned}
$$

where

$$
\begin{aligned}
& M(\zeta)=8\left(C_{11} \beta_{1} \gamma_{3}+C_{12}\right)\left(C_{11} \beta_{2} \gamma_{4}+C_{12}\right)\left(\zeta^{-1} \gamma_{3}-\zeta \beta_{1}\right)\left(\zeta^{-1} \gamma_{4}\right. \\
& \left.-\zeta \beta_{2}\right)\left\{\cosh \left(\gamma_{3} h\right) \cosh \left(\gamma_{4} h\right)-1\right\}-4\left\{\left(\zeta^{-1} \gamma_{3}-\zeta \beta_{1}\right)^{2}\left(C_{11} \beta_{2} \gamma_{4}+C_{12}\right)^{2}\right. \\
& \left.+\left(\zeta^{-1} \gamma_{4}-\zeta \beta_{2}\right)^{2}\left(C_{11} \beta_{1} \gamma_{3}+C_{12}\right)^{2}\right\} \sinh \left(\gamma_{3} h\right) \sinh \left(\gamma_{4} h\right) \\
& q_{1}(\zeta)=-\frac{2 \zeta}{\pi} \int_{-\infty}^{\infty}\left[\frac{i \alpha_{1}-\gamma_{1}}{\zeta^{2}+\gamma_{1}^{2}}+\frac{\beta\left(i \alpha_{2}-\gamma_{2}\right)}{\zeta^{2}+\gamma_{2}^{2}}\right] \frac{\xi A(\xi)}{\alpha_{1}+\beta \alpha_{2}} d \xi \\
& q_{2}(\zeta)=-\frac{2 \zeta}{\pi} \int_{-\infty}^{\infty}\left[\frac{i \alpha_{1}-\gamma_{1}}{\zeta^{2}+\gamma_{1}^{2}}+\frac{\beta\left(i \alpha_{2}-\gamma_{2}\right)}{\zeta^{2}+\gamma_{2}^{2}}\right] \frac{\xi A(\xi) e^{i \xi h}}{\alpha_{1}+\beta \alpha_{2}} d \xi \\
& q_{3}(\zeta)=-\frac{2}{\pi} \int_{-\infty}^{\infty}\left[\frac{\gamma_{1}\left(i \xi^{2} C_{11}-\alpha_{1} \gamma_{1} C_{12}\right)}{\zeta^{2}+\gamma_{1}^{2}}+\frac{\gamma_{2} \beta\left(i \xi^{2} C_{11}-\alpha_{2} \gamma_{2} C_{12}\right)}{\zeta_{1}^{2}+\beta \alpha_{2}} d \xi\right. \\
& q_{4}(\zeta)=-\frac{2}{\pi} \int_{-\infty}^{\infty}\left[\frac{\gamma_{1}\left(i \xi^{2} C_{11}-\alpha_{1} \gamma_{1} C_{12}\right)}{\zeta^{2}+\gamma_{1}^{2}}+\frac{\gamma_{2} \beta\left(i \xi^{2} C_{11}-\alpha_{2} \gamma_{2} C_{12}\right)}{\zeta^{2}+\gamma_{2}^{2}}\right] \frac{A(\xi) e^{i \xi h}}{\alpha_{1}+\beta \alpha_{2}} d \xi
\end{aligned}
$$

Next, substituting the value of $A(\xi)$ from Eq. (27) in the expressions of $E_{1}(\zeta), E_{2}(\zeta)$, $E_{3}(\zeta)$ and $E_{4}(\zeta)$, the Eq. (31) reduces to

$$
R(t)=-\frac{\mu_{12}^{-1} p_{0}}{a_{0}}-\int_{0}^{1} u g(u)\left[L_{2}(u, t)+L_{3}(u, t)+L_{4}(u, t)\right] d u
$$

where

$$
\begin{aligned}
L_{2}(u, t)=- & \frac{1}{\pi \theta} \int_{0}^{\infty} \zeta F_{1}(\zeta, t) \int_{-\infty}^{\infty}\left[\frac{i \alpha_{1}-\gamma_{1}}{\zeta^{2}+\gamma_{1}^{2}}+\frac{\beta\left(i \alpha_{2}-\gamma_{2}\right)}{\zeta^{2}+\gamma_{2}^{2}}\right] \frac{\xi J_{0}(\xi u) e^{i \xi h}}{\alpha_{1}+\beta \alpha_{2}} d \xi d \zeta \\
L_{3}(u, t)=- & \frac{1}{\pi \theta} \int_{0}^{\infty} F_{2}(\zeta, t) \int_{-\infty}^{\infty}\left[\frac{\gamma_{1}\left(i \xi^{2} C_{11}-\alpha_{1} \gamma_{1} C_{12}\right)}{\zeta^{2}+\gamma_{1}^{2}}+\frac{\gamma_{2} \beta\left(i \xi^{2} C_{11}-\alpha_{2} \gamma_{2} C_{12}\right)}{\zeta^{2}+\gamma_{2}^{2}}\right] \\
& \frac{J_{0}(\xi u)}{\alpha_{1}+\beta \alpha_{2}} d \xi d \zeta \\
L_{4}(u, t)=- & \frac{1}{\pi \theta} \int_{0}^{\infty} F_{3}(\zeta, t) \int_{-\infty}^{\infty}\left[\frac{\gamma_{1}\left(i \xi^{2} C_{11}-\alpha_{1} \gamma_{1} C_{12}\right)}{\zeta^{2}+\gamma_{1}^{2}}+\frac{\gamma_{2} \beta\left(i \xi^{2} C_{11}-\alpha_{2} \gamma_{2} C_{12}\right)}{\zeta^{2}+\gamma_{2}^{2}}\right] \\
& \frac{J_{0}(\xi u) e^{i \xi h}}{\alpha_{1}+\beta \alpha_{2}} d \xi d \zeta
\end{aligned}
$$


and

$$
\begin{aligned}
& M(\zeta) F_{1}(\zeta, t)=4\left(C_{12} \beta_{1} \gamma_{3}+C_{22}\right)\left(C_{11} \beta_{2} \gamma_{4}+C_{12}\right)\left[( C _ { 1 1 } \beta _ { 1 } \gamma _ { 3 } + C _ { 1 2 } ) ( \gamma _ { 4 } \zeta ^ { - 1 } - \zeta \beta _ { 2 } ) \left\{\sinh \left(\gamma_{3} h\right) I_{0}\left(\gamma_{3} t\right)\right.\right. \\
& \left.\left.-\left(\cosh \left(\gamma_{3} h\right)-\cosh \left(\gamma_{4} h\right)\right) L_{0}\left(\gamma_{3} t\right)\right\}-\left(C_{11} \beta_{2} \gamma_{4}+C_{12}\right)\left(\gamma_{3} \zeta^{-1}-\zeta \beta_{1}\right) \sinh \left(\gamma_{4} h\right) I_{0}\left(\gamma_{3} t\right)\right] \\
& -4\left(C_{12} \beta_{2} \gamma_{4}+C_{22}\right)\left(C_{11} \beta_{1} \gamma_{3}+C_{12}\right)\left[\left(C_{11} \beta_{1} \gamma_{3}+C_{12}\right)\left(\gamma_{4} \zeta^{-1}-\zeta \beta_{2}\right) \sinh \left(\gamma_{3} h\right) I_{0}\left(\gamma_{4} t\right)\right. \\
& -\left(C_{11} \beta_{2} \gamma_{4}+C_{12}\right)\left(\gamma_{3} \zeta^{-1}-\zeta \beta_{1}\right)\left\{\sinh \left(\gamma_{4} h\right) I_{0}\left(\gamma_{4} t\right)\right. \\
& \left.\left.-\left(\cosh \left(\gamma_{3} h\right)-\cosh \left(\gamma_{4} h\right)\right) L_{0}\left(\gamma_{4} t\right)\right\}\right] \\
& M(\zeta) F_{2}(\zeta, t)=4\left(C_{12} \beta_{1} \gamma_{3}+C_{22}\right)\left(C_{11} \beta_{1} \gamma_{3}+C_{12}\right)\left(\gamma_{4} \zeta^{-1}-\zeta \beta_{2}\right)^{2} \sinh \left(\gamma_{4} h\right) \\
& \left.\left.\left\{\cosh \left(\gamma_{3} h\right)\right) L_{0}\left(\gamma_{3} t\right)-\sinh \left(\gamma_{3} h\right)\right) I_{0}\left(\gamma_{3} t\right)\right\} \\
& -4\left(C_{12} \beta_{1} \gamma_{3}+C_{22}\right)\left(C_{11} \beta_{2} \gamma_{4}+C_{12}\right)\left(\gamma_{4} \zeta^{-1}-\zeta \beta_{2}\right)\left(\gamma_{3} \zeta^{-1}-\zeta \beta_{1}\right) \\
& {\left[\left\{1-\cosh \left(\gamma_{3} h\right) \cosh \left(\gamma_{4} h\right)\right\} I_{0}\left(\gamma_{3} t\right)+\cosh \left(\gamma_{4} h\right) \sinh \left(\gamma_{3} h\right) L_{0}\left(\gamma_{3} t\right)\right]} \\
& +4\left(C_{12} \beta_{2} \gamma_{4}+C_{22}\right)\left(C_{11} \beta_{2} \gamma_{4}+C_{12}\right)\left(\gamma_{3} \zeta^{-1}-\zeta \beta_{1}\right)^{2} \sinh \left(\gamma_{3} h\right) \\
& \left.\left.\left\{\cosh \left(\gamma_{4} h\right)\right) L_{0}\left(\gamma_{4} t\right)-\sinh \left(\gamma_{4} h\right)\right) I_{0}\left(\gamma_{4} t\right)\right\} \\
& -4\left(C_{12} \beta_{2} \gamma_{4}+C_{22}\right)\left(C_{11} \beta_{1} \gamma_{3}+C_{12}\right)\left(\gamma_{4} \zeta^{-1}-\zeta \beta_{2}\right)\left(\gamma_{3} \zeta^{-1}-\zeta \beta_{1}\right) \\
& {\left[\left\{1-\cosh \left(\gamma_{3} h\right) \cosh \left(\gamma_{4} h\right)\right\} I_{0}\left(\gamma_{4} t\right)+\cosh \left(\gamma_{3} h\right) \sinh \left(\gamma_{4} h\right) L_{0}\left(\gamma_{4} t\right)\right]} \\
& M(\zeta) F_{3}(\zeta, t)=-4\left(C_{12} \beta_{1} \gamma_{3}+C_{22}\right)\left(C_{11} \beta_{1} \gamma_{3}+C_{12}\right)\left(\gamma_{4} \zeta^{-1}-\zeta \beta_{2}\right)^{2} \sinh \left(\gamma_{4} h\right) L_{0}\left(\gamma_{3} t\right) \\
& -4\left(C_{12} \beta_{2} \gamma_{4}+C_{22}\right)\left(C_{11} \beta_{2} \gamma_{4}+C_{12}\right)\left(\gamma_{3} \zeta^{-1}-\zeta \beta_{1}\right)^{2} \sinh \left(\gamma_{3} h\right) L_{0}\left(\gamma_{4} t\right) \\
& +4\left(C_{12} \beta_{1} \gamma_{3}+C_{22}\right)\left(C_{11} \beta_{2} \gamma_{4}+C_{12}\right)\left(\gamma_{4} \zeta^{-1}-\zeta \beta_{2}\right)\left(\gamma_{3} \zeta^{-1}-\zeta \beta_{1}\right) \\
& {\left[\left\{\cosh \left(\gamma_{4} h\right)-\cosh \left(\gamma_{3} h\right)\right\} I_{0}\left(\gamma_{3} t\right)+\sinh \left(\gamma_{3} h\right) L_{0}\left(\gamma_{3} t\right)\right]} \\
& +4\left(C_{12} \beta_{2} \gamma_{4}+C_{22}\right)\left(C_{11} \beta_{1} \gamma_{3}+C_{12}\right)\left(\gamma_{4} \zeta^{-1}-\zeta \beta_{2}\right)\left(\gamma_{3} \zeta^{-1}-\zeta \beta_{1}\right) \\
& {\left[\left\{\cosh \left(\gamma_{3} h\right)-\cosh \left(\gamma_{4} h\right)\right\} I_{0}\left(\gamma_{4} t\right)+\sinh \left(\gamma_{4} h\right) L_{0}\left(\gamma_{4} t\right)\right]}
\end{aligned}
$$

and $I_{0}()$ is the modified Bessel function of imaginary argument of order zero and $L_{0}()$ is the modified Struve function of order zero.

Now substituting $R(t)$ from the Eq. (41) and $g_{1}(t)=-\frac{a_{0} \mu_{12}}{p_{0}} g(t)$ in Eq. (29), we finally obtain the following Fredholm Integral equation

$$
g_{1}(t)+\int_{0}^{1} u g_{1}(u) L(u, t) d u=1
$$

where

$$
L(u, t)=L_{1}(u, t)+L_{2}(u, t)+L_{3}(u, t)+L_{4}(u, t)
$$

It is to be noted that the kernel $L_{1}(u, t)$ represented by the semi-infinite integral given by the Eq. (30) has a slow rate of convergence. In order to make the numerical analysis easier, the semi-infinite integral has therefore been converted to finite integrals by using simple contour integration technique [16] and is given by

$$
\begin{aligned}
L_{1}(u, t)= & -i \int_{0}^{\frac{k_{s}}{\sqrt{C_{11}}}}\left[\frac{\left(\xi^{2} C_{12}-C_{22} \gamma_{1}^{\prime} \alpha_{1}^{\prime}\right)+\beta^{\prime}\left(\xi^{2} C_{12}-C_{22} \gamma_{2}^{\prime} \alpha_{2}^{\prime}\right)}{\theta\left(\alpha_{1}^{\prime}+\beta^{\prime} \alpha_{2}^{\prime}\right)}\right] J_{0}(\xi u) H_{0}^{(1)}(\xi t) d \xi \\
& -i \int_{\frac{k_{s}}{\sqrt{C_{11}}}}^{k_{s}} \frac{\beta^{\prime \prime}\left(\xi^{2} C_{12}-C_{22} \gamma_{2}^{\prime \prime} \alpha_{2}^{\prime \prime}\right)}{\theta\left(\alpha_{1}^{\prime \prime}+\beta^{\prime \prime} \alpha_{2}^{\prime \prime}\right)} J_{0}(\xi u) H_{0}^{(1)}(\xi t) d \xi, \quad(t>u)
\end{aligned}
$$

where

$$
\alpha_{j}^{\prime}=\frac{\gamma_{j}^{\prime 2}+C_{11} \xi^{2}-k_{s}^{2}}{\gamma_{j}^{\prime}\left(1+C_{12}\right)} \quad(j=1,2)
$$




$$
\begin{aligned}
& \beta^{\prime}=\frac{\alpha_{1}^{\prime}-\gamma_{1}^{\prime}}{\gamma_{2}^{\prime}-\alpha_{2}^{\prime}} \\
& \alpha_{1}^{\prime \prime}=\frac{\gamma_{1}^{\prime \prime 2}-C_{11} \xi^{2}+k_{s}^{2}}{\gamma_{1}^{\prime \prime}\left(1+C_{12}\right)} \\
& \alpha_{2}^{\prime \prime}=\frac{\gamma_{2}^{\prime \prime 2}+C_{11} \xi^{2}-k_{s}^{2}}{\gamma_{2}^{\prime \prime}\left(1+C_{12}\right)} \\
& \beta^{\prime \prime}=\frac{\alpha_{1}^{\prime \prime}-\gamma_{1}^{\prime \prime}}{\gamma_{2}^{\prime \prime}-\alpha_{2}^{\prime \prime}} \\
& \gamma_{1}^{\prime}=\sqrt{\frac{1}{2}\left(B_{1}-\sqrt{B_{1}^{2}-4 B_{2}^{\prime}}\right)} \\
& \gamma_{2}^{\prime}=\sqrt{\frac{1}{2}\left(B_{1}+\sqrt{B_{1}^{2}-4 B_{2}^{\prime}}\right)} \\
& B_{1}=\frac{1}{C_{22}}\left[\left(1+C_{22}\right) k_{s}^{2}+\left(C_{12}^{2}+2 C_{12}-C_{11} C_{22}\right) \xi^{2}\right] \\
& B_{2}^{\prime}=\frac{C_{11}}{C_{22}}\left(k_{s}^{2}-\xi^{2}\right)\left(\frac{k_{s}^{2}}{C_{11}}-\xi^{2}\right) \\
& \gamma_{1}^{\prime \prime}=\sqrt{\frac{1}{2}\left(-B_{1}+\sqrt{B_{1}^{2}+4 B_{2}^{\prime \prime}}\right)} \\
& \gamma_{2}^{\prime \prime}=\sqrt{\frac{1}{2}\left(B_{1}+\sqrt{B_{1}^{2}+4 B_{2}^{\prime \prime}}\right)} \\
& B_{2}^{\prime}=-B_{2}^{\prime \prime}
\end{aligned}
$$

and $H_{0}^{(1)}$ is the Hankel function.

The corresponding expression of $L_{1}(u, t)$ for $u>t$ is obtained by interchanging $\mathrm{u}$ and $\mathrm{t}$ in Eq. (50).

\section{Stress Intensity Factor and Stress Outside the Crack}

The normal stress $\sigma_{y y}(x, y)$ in the plane $y=0$ in the neighbourhood of the crack can be found from the Eq. (23) and is given by

$$
\begin{aligned}
\sigma_{y y}(x, 0)= & \mu_{12} \int_{-\infty}^{\infty}\left[\left(i \xi^{2} C_{12}-\alpha_{1} \gamma_{1} C_{22}\right)+\beta\left(i \xi^{2} C_{12}-\alpha_{2} \gamma_{2} C_{22}\right)\right] \frac{A(\xi) e^{i \xi x}}{\alpha_{1}+\beta \alpha_{2}} d \xi \\
& +\mu_{12} \int_{0}^{\infty}\left[\left(C_{12} \beta_{1} \gamma_{3}+C_{22}\right)\left\{E_{1}(\zeta) e^{\gamma_{3} x}+E_{2}(\zeta) e^{-\gamma_{3} x}\right\}\right. \\
& \left.+\left(C_{12} \beta_{2} \gamma_{4}+C_{22}\right)\left\{E_{3}(\zeta) e^{\gamma_{4} x}+E_{4}(\zeta) e^{-\gamma_{4} x}\right\}\right] d \zeta
\end{aligned}
$$

Substituting the values of $A(\xi), E_{1}(\zeta), E_{2}(\zeta), E_{3}(\zeta)$ and $E_{4}(\zeta)$, the expression for the stress can finally be represented as

$$
\sigma_{y y}(x, 0)=\frac{p_{0} x}{\sqrt{x^{2}-1}} g_{1}(1)+O(1) \quad(x>1)
$$


Defining stress intensity factor $\mathrm{K}$ by

$$
K=\lim _{x \rightarrow 1+}\left|\frac{\sqrt{x-1} \sigma_{y y}(x, 0)}{p_{0}}\right|
$$

we obtain

$$
K=\frac{\left|g_{1}(1)\right|}{\sqrt{2}}
$$

Now from the Eq. (23), after substituting the value of $A(\xi), E_{1}(\zeta), E_{2}(\zeta), E_{3}(\zeta)$ and $E_{4}(\zeta)$, we have

$$
\begin{aligned}
\sigma_{y y}(x, y)= & -\frac{p_{0}}{\theta} \int_{0}^{1} u g_{1}(u) \int_{0}^{\infty}\left[\left(i \xi^{2} C_{12}-\alpha_{1} \gamma_{1} C_{22}\right) e^{-\gamma_{1} y}\right. \\
& \left.+\beta e^{-\gamma_{2} y}\left(i \xi^{2} C_{12}-\alpha_{2} \gamma_{2} C_{22}\right)\right] \frac{J_{0}(\xi u) \cos (\xi x)}{\alpha_{1}+\beta \alpha_{2}} d \xi d u \\
& -\frac{p_{0}}{\theta} \int_{0}^{1} u g_{1}(u)\left[R_{1}(u, x, y)+R_{2}(u, x, y)+R_{3}(u, x, y)\right] d u
\end{aligned}
$$

where

$$
\begin{aligned}
R_{1}(u, x, y)=- & \frac{1}{\pi} \int_{0}^{\infty} \zeta D(\zeta, x, y) \int_{-\infty}^{\infty}\left[\frac{i \alpha_{1}-\gamma_{1}}{\zeta^{2}+\gamma_{1}^{2}}\right. \\
& \left.+\frac{\beta\left(i \alpha_{2}-\gamma_{2}\right)}{\zeta^{2}+\gamma_{2}^{2}}\right] \frac{\xi J_{0}(\xi u) e^{i \xi h}}{\alpha_{1}+\beta \alpha_{2}} d \xi d \zeta \\
R_{2}(u, x, y)=- & \frac{1}{\pi} \int_{0}^{\infty} E(\zeta, x, y) \int_{-\infty}^{\infty}\left[\frac{\gamma_{1}\left(i \xi^{2} C_{11}-\alpha_{1} \gamma_{1} C_{12}\right)}{\zeta^{2}+\gamma_{1}^{2}}\right. \\
& \left.+\frac{\gamma_{2} \beta\left(i \xi^{2} C_{11}-\alpha_{2} \gamma_{2} C_{12}\right)}{\zeta^{2}+\gamma_{2}^{2}}\right] \frac{J_{0}(\xi u)}{\alpha_{1}+\beta \alpha_{2}} d \xi d \zeta \\
R_{3}(u, x, y)=- & \frac{1}{\pi} \int_{0}^{\infty} F(\zeta, x, y) \int_{-\infty}^{\infty}\left[\frac{\gamma_{1}\left(i \xi^{2} C_{11}-\alpha_{1} \gamma_{1} C_{12}\right)}{\zeta^{2}+\gamma_{1}^{2}}\right. \\
& \left.+\frac{\gamma_{2} \beta\left(i \xi^{2} C_{11}-\alpha_{2} \gamma_{2} C_{12}\right)}{\zeta^{2}+\gamma_{2}^{2}}\right] \frac{J_{0}(\xi u) e^{i \xi h}}{\alpha_{1}+\beta \alpha_{2}} d \xi d \zeta
\end{aligned}
$$

and

$$
\begin{aligned}
& D(\zeta, x, y)=\left[( C _ { 1 2 } \beta _ { 1 } \gamma _ { 3 } + C _ { 2 2 } ) ( C _ { 1 1 } \beta _ { 2 } \gamma _ { 4 } + C _ { 1 2 } ) \left\{-\left(\zeta^{-1} \gamma_{3}-\zeta \beta_{1}\right)\left(C_{11} \beta_{2} \gamma_{4}\right.\right.\right. \\
& \left.\quad+C_{12}\right) \sinh \left(\gamma_{4} h\right) \cosh \left(\gamma_{3} x\right) \\
& \left.\quad+\left(\zeta^{-1} \gamma_{4}-\zeta \beta_{2}\right)\left(C_{11} \beta_{1} \gamma_{3}+C_{12}\right)\left(\cosh \left(\gamma_{4} h\right) \sinh \left(\gamma_{3} x\right)-\sinh \left(\gamma_{3}(x-h)\right)\right)\right\} \\
& \quad+\left(C_{12} \beta_{2} \gamma_{4}+C_{22}\right)\left(C_{11} \beta_{1} \gamma_{3}+C_{12}\right)\left\{-\left(\zeta^{-1} \gamma_{4}-\zeta \beta_{2}\right)\left(C_{11} \beta_{1} \gamma_{3}\right.\right. \\
& \left.\quad+C_{12}\right) \sinh \left(\gamma_{3} h\right) \cosh \left(\gamma_{4} x\right) \\
& \quad+\left(\zeta^{-1} \gamma_{3}-\zeta \beta_{1}\right)\left(C_{11} \beta_{2} \gamma_{4}+C_{12}\right)\left(\sinh \left(\gamma_{4} x\right) \cosh \left(\gamma_{3} h\right)\right. \\
& \left.\left.\left.\quad-\sinh \left(\gamma_{4}(x-h)\right)\right)\right\}\right] \frac{4 \cos (\zeta y)}{M(\zeta)}
\end{aligned}
$$

$E(\zeta, x, y)=\left[\left(C_{12} \beta_{1} \gamma_{3}+C_{22}\right)\left(\zeta^{-1} \gamma_{4}-\zeta \beta_{2}\right)\left\{\left(\zeta^{-1} \gamma_{4}-\zeta \beta_{2}\right)\left(C_{11} \beta_{1} \gamma_{3}\right.\right.\right.$

$\left.+C_{12}\right) \sinh \left(\gamma_{4} h\right) \sinh \left(\gamma_{3}(x-h)\right)$ 


$$
\begin{aligned}
&+\left.\left(\zeta^{-1} \gamma_{3}-\zeta \beta_{1}\right)\left(C_{11} \beta_{2} \gamma_{4}+C_{12}\right)\left(\cosh \left(\gamma_{4} h\right) \cosh \left(\gamma_{3}(x-h)\right)-\cosh \left(\gamma_{3} x\right)\right)\right\} \\
&+\left(C_{12} \beta_{2} \gamma_{4}+C_{22}\right)\left(\zeta^{-1} \gamma_{3}-\zeta \beta_{1}\right)\left\{( \zeta ^ { - 1 } \gamma _ { 3 } - \zeta \beta _ { 1 } ) \left(C_{11} \beta_{2} \gamma_{4}\right.\right. \\
&+\left.C_{12}\right) \sinh \left(\gamma_{4}(x-h)\right) \sinh \left(\gamma_{3} h\right) \\
&+\left(\zeta^{-1} \gamma_{4}-\zeta \beta_{2}\right)\left(C_{11} \beta_{1} \gamma_{3}+C_{12}\right)\left(\cosh \left(\gamma_{3} h\right) \cosh \left(\gamma_{4}(x-h)\right)\right. \\
&\left.\left.\left.+\cosh \left(\gamma_{4} x\right)\right)\right\}\right] \frac{4 \cos (\zeta y)}{M(\zeta)} \\
& F(\zeta, x, y)=\left[( C _ { 1 2 } \beta _ { 1 } \gamma _ { 3 } + C _ { 2 2 } ) ( \zeta ^ { - 1 } \gamma _ { 4 } - \zeta \beta _ { 2 } ) \left\{-\left(\zeta^{-1} \gamma_{4}-\zeta \beta_{2}\right)\left(C_{11} \beta_{1} \gamma_{3}\right.\right.\right. \\
&\left.+C_{12}\right) \sinh \left(\gamma_{4} h\right) \sinh \left(\gamma_{3} x\right) \\
&\left.+\left(\zeta^{-1} \gamma_{3}-\zeta \beta_{1}\right)\left(C_{11} \beta_{2} \gamma_{4}+C_{12}\right)\left(\cosh \left(\gamma_{3} x\right) \cosh \left(\gamma_{4} h\right)-\cosh \left(\gamma_{3}(x-h)\right)\right)\right\} \\
&+\left(C_{12} \beta_{2} \gamma_{4}+C_{22}\right)\left(\zeta^{-1} \gamma_{3}-\zeta \beta_{1}\right)\left\{-\left(\zeta^{-1} \gamma_{3}-\zeta \beta_{1}\right)\left(C_{11} \beta_{2} \gamma_{4}+C_{12}\right) \sinh \left(\gamma_{4} x\right) \sinh \left(\gamma_{3} h\right)\right. \\
&+\left(\zeta^{-1} \gamma_{4}-\zeta \beta_{2}\right)\left(C_{11} \beta_{1} \gamma_{3}+C_{12}\right)\left(\cosh \left(\gamma_{4} x\right) \cosh \left(\gamma_{3} h\right)\right. \\
&\left.\left.+\cosh \left(\gamma_{4}(x-h)\right)\right)\right\} \frac{4 \cos (\zeta y)}{M(\zeta)}
\end{aligned}
$$

\section{Numerical Results and Discussions}

The integral Eq. (48) has been solved numerically by the method of Fox and Goodwin [17] for different values of parameters $k_{s}$ and $h$. The integral in (48) has been represented by a quadrature formula involving values of the specified range of integration, and then converted to a set of simultaneous linear algebraic equations. The solution of linear algebraic equations

Table 1 Engineering elastic constants

\begin{tabular}{llccc}
\hline & & $C_{11}$ & $C_{22}$ & $C_{12}$ \\
\hline Type I & E-type glass-epoxi composite & 2.721 & 11.759 & 0.741 \\
Type II & Steel-Mylar composite & 18.7 & 2.92 & 1.3 \\
\hline
\end{tabular}

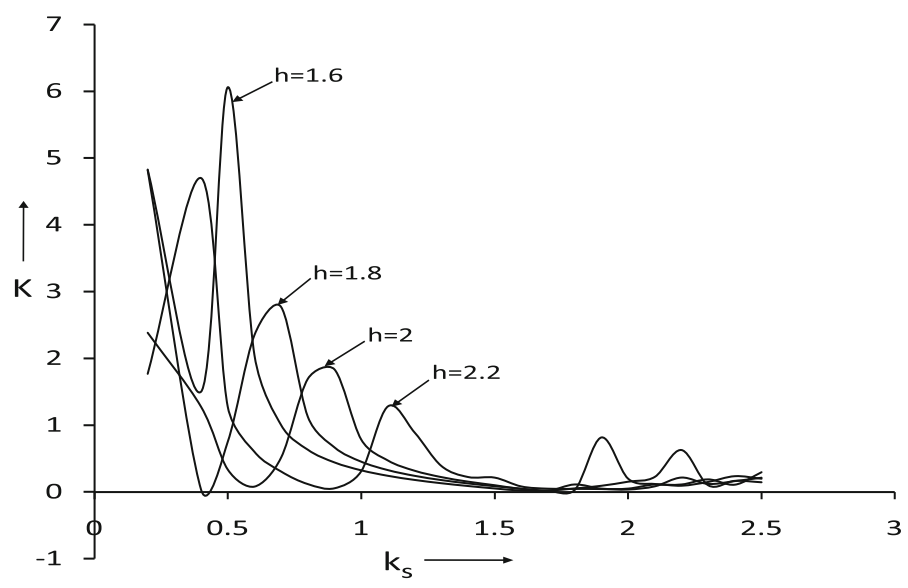

Fig. 2 Stress intensity factor $\mathrm{K}$ versus dimensionless frequency $k_{s}$ for Type I 


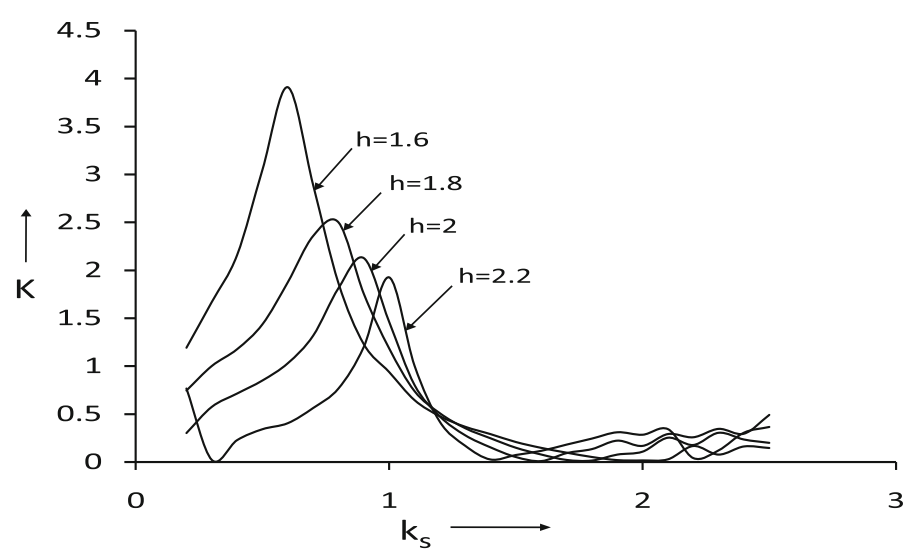

Fig. 3 Stress intensity factor $\mathrm{K}$ versus dimensionless frequency $k_{s}$ for Type II

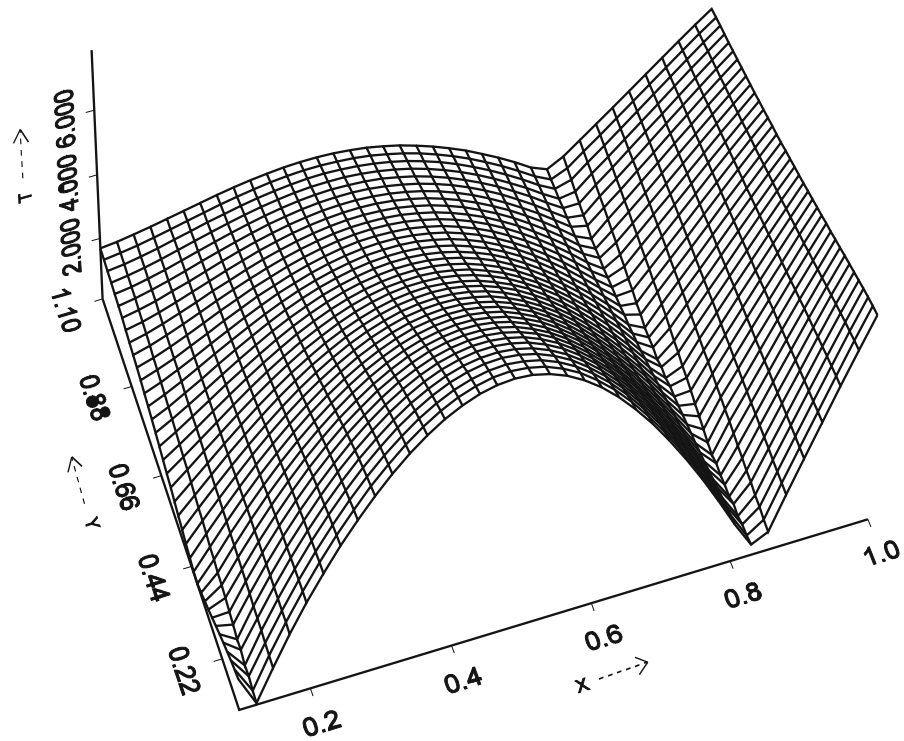

Fig. 4 Normal stress T outside the crack for Type I $\left(h=2.5, k_{s}=0.1\right)$

gives a first approximation to the pivotal values of $g_{1}(t)$ which has been improved by the use of difference correction technique.

After solving the integral Eq. (48), the SIF has been calculated numerically for different values of $h$ and plotted against frequency $k_{s}$ for two different types of orthotropic materials whose engineering constants have been listed in the following Table 1:

Figures 2 and 3 show the variations of $\operatorname{SIF}(\mathrm{K})$ with dimensionless frequency $\mathrm{k}_{s}$ for various values of $\mathrm{h}(1.6,1.8,2.0,2.2)$ for type I and type II materials respectively. It is observed that $\mathrm{K}$ initially increases and after obtaining maximum value, again it decreases with the increase in the values of $\mathrm{k}_{s}$. Also it is noted that $\mathrm{K}$ becomes lower and more flatten as strip width $\mathrm{h}$ increases which is physically consistent. 


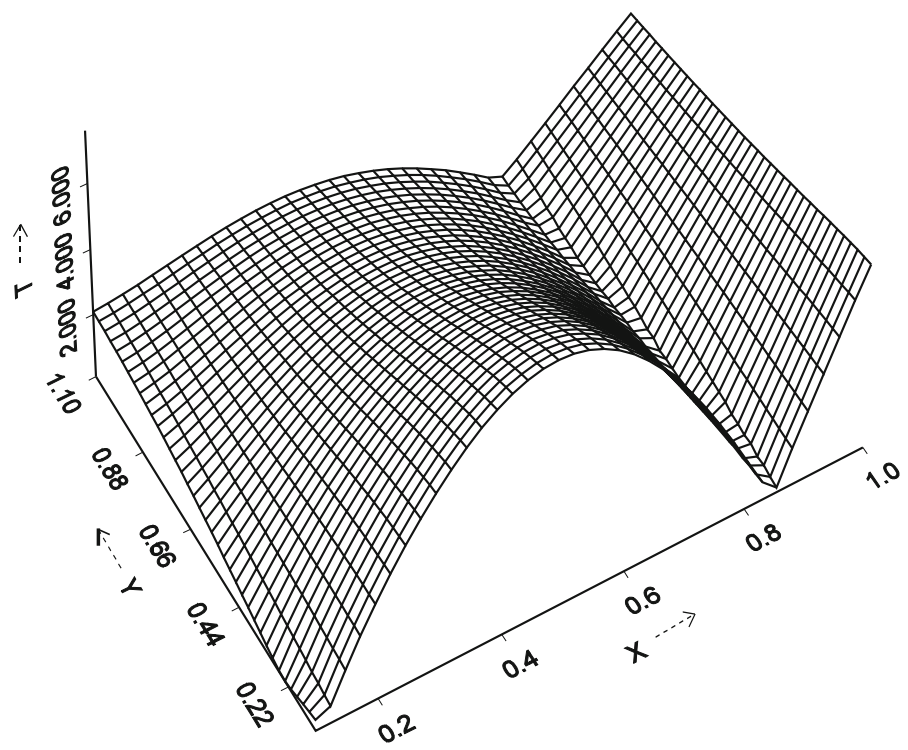

Fig. 5 Normal stress T outside the crack for Type I $\left(h=2.6, k_{s}=0.1\right)$

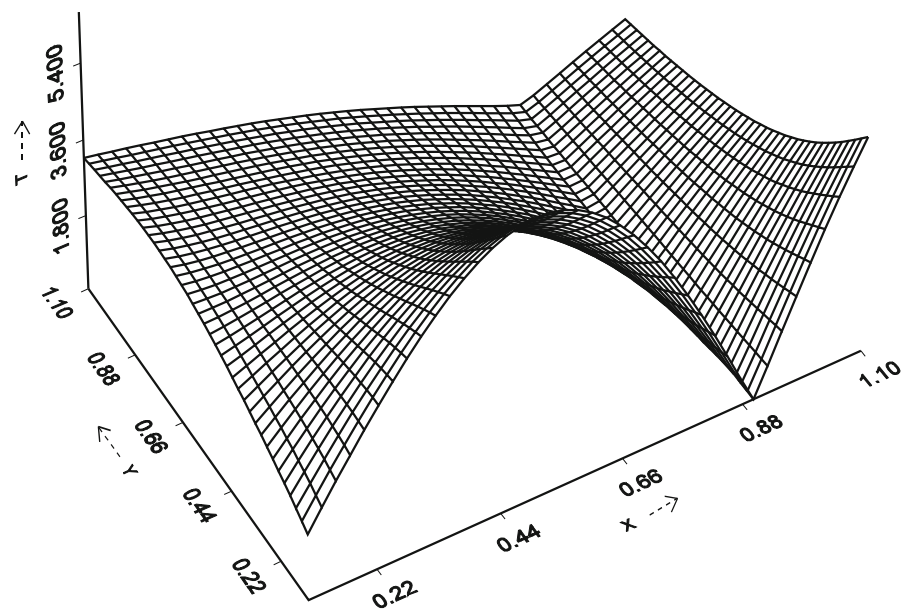

Fig. 6 Normal stress T outside the crack for Type II $\left(h=2.5, k_{s}=0.1\right)$

Next, dimensionless normal stress $T=\sigma_{y y}(x, y) / p_{0}$, at distant points from the crack has been computed numerically from Eq. (54) and presented by means of 3-dimensional graphs (Figs. $4,5,6,7)$ for various values of $\mathrm{h}(2.5,2.6)$ for type I and type II materials. The influence of the strip width $\mathrm{h}$ and material properties are shown in the graphs.

\section{Conclusion}

From the numerical results it is observed that influence of SIF can be controlled by varying strip width. Also the effect of material orthotropy on SIF and normal stress is significant. 


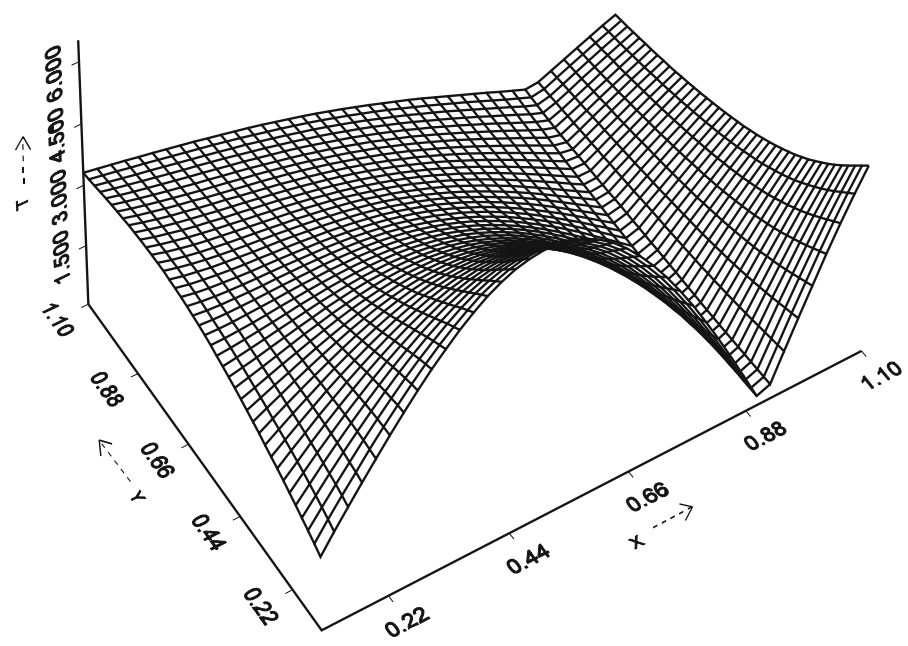

Fig. 7 Normal stress T outside the crack for Type II $\left(h=2.6, k_{s}=0.1\right)$

Acknowledgments This research work is supported by the Project-Mobile Computing and Innovative Applications under UPE-II Programme of Jadavpur University, Kolkata and The Department of Science and Technology(DST), New Delhi, India under The INSPIRE Programme.

\section{References}

1. Cinar, A., Erdogan, F.: The crack and wedging problem for an orthotropic strip. Int. J. Fract. 19, 83-102 (1983)

2. Satapathy, P.K., Parhi, H.: Stresses in an orthotropic strip containing a griffith crack. Int. J. Eng. Sci. 16, 147-154 (1978)

3. Piva, A., Viola, E.: Crack propagation in an orthotropic medium. Eng. Fract. Mech. 29(5), 535-548 (1988)

4. Kassir, M.K., Tse, S.: Moving griffith crack in an orthotropic material. Int. J. Eng. Sci. 21(4), 315-325 (1983)

5. Dhaliwal, R.S.: Two coplanar cracks in an infinitely long orthotropic elastic strip. Utilitas Math. 4, 115128 (1973)

6. Das, S., Debnath, L.: Interaction between griffith cracks in a sandwiched orthotropic layer. Appl. Math. Lett. 16(4), 609-617 (2003)

7. Chen, E.P.: Sudden appearance of a crack in a stretched finite strip. J. Appl. Mech. 45(2), 277-280 (1978)

8. Srivastava, K.N., Gupta, O.P., Palaiya, R.M.: Interaction of elastic waves with a griffith cracks situated in an infinitely long strip. J. Appl. Math. Mech. 61(11), 583-587 (1981)

9. Shindo, Y., Nozaki, H., Higaki, H.: Impact response of a finite crack in an orthotropic strip. Acta Mech. 62(1-4), 87-104 (1986)

10. Sarkar, J., Mandal, S.C., Ghosh, M.L.: Diffraction of elastic waves by three coplaner griffith cracks in an orthotropic medium. Int. J. Eng. Sci. 33(2), 163-177 (1995)

11. Munshi, N., Mandal, S.C.: Diffraction of p-waves by edge crack in an infinitely long elastic strip. JSME Int. J. Ser. A 49(1), 116-122 (2006)

12. Das, S., Chakraborty, S., Srikanth, N., Gupta, M.: Symmetric edge cracks in an orthotropic strip under normal loading. Int. J. Fract. 153(1), 77-84 (2008)

13. Das, S.: Weight function for an edge crack in an infinite orthotropic strip under normal point loading. J. Appl. Math. Mech. 90(4), 271-277 (2010)

14. Das, S., Mukhopadhyay, S., Prasad, R.: Stress intensity factor of an edge crack in bonded orthotropic materials. Int. J. Fract. 168(1), 117-123 (2011)

15. Das, S., Prasad, R., Mukhopadhyay, S.: Stress intensity factor of an edge crack in composite media. Int. J. Fract. 172(2), 201-207 (2011) 
16. Mandal, S.C., Ghosh, M.L.: Interaction of elastic waves with a periodic array of coplaner griffith crack in an orthotropic medium. Int. J. Eng. Sci. 32(1), 167-178 (1994)

17. Fox, L., Goodwin, E.T.: The numerical solution of non-singular integral equations. Philos. Trans. R. Soc. Lond. Ser. A 245, 501-534 (1953) 